\title{
Evolving target volume concepts in locally advanced non-small cell lung cancer
}

\author{
Ursula Nestle $e^{1,2,3}$, Cecile Le Pechoux ${ }^{4}$, Dirk De Ruysscher ${ }^{5}$ \\ ${ }^{1}$ Department of Radiation Oncology, University of Freiburg, Medical Center Faculty of Medicine, Freiburg, Germany; ${ }^{2}$ German Cancer Consortium \\ (DKTK) Partner Site Freiburg and German Cancer Research Center (DKFZ), Heidelberg, Germany; ${ }^{3}$ Department of Radiation Oncology, Kliniken \\ Maria Hilf, Mönchengladbach, Germany; ${ }^{4}$ Department of Radiation Oncology, Gustave Roussy, Institut d'Oncologie Thoracique (IOT), Villejuif, \\ France; ${ }^{5}$ Department of Radiation Oncology (Maastro Clinic), Maastricht University Medical Center+, GROW Research Institute, Maastricht, The \\ Netherlands \\ Contributions: (I) Conception and design: U Nestle; (II) Administrative support: None; (III) Provision of study materials or patients: None; (IV) \\ Collection and assembly of data: None; (V) Data analysis and interpretation: None; (VI) Manuscript writing: All authors; (VII) Final approval of \\ manuscript: All authors. \\ Correspondence to: Prof. Dr. med Ursula Nestle. Kliniken Maria Hilf, Viersener Str. 450, 41063 Mönchengladbach, Germany. \\ Email: Ursula.Nestle@mariahilf.de.
}

\begin{abstract}
Radiotherapy (RT) target volume concepts for locally advanced lung cancer have been under discussion for years. Although they may be as important as treatment doses, many aspects of them are still based on conventions, which, due to the paucity of prospective data, rely on long-term practice or on clinical knowledge and experience (e.g., on patterns of spread or recurrence). However, in recent years, large improvements have been made in medical imaging and molecular imaging methods have been implemented, which are of great interest in RT. For lung cancer, in recent years, 18F-fluoro-desoxy-glucose (FDG)positron-emission tomography (PET)/computed tomography (CT) has shown a superior diagnostic accuracy as compare to conventional imaging and has become an indispensable standard tool for diagnostic workup, staging and response assessment. This offers the chance to optimize target volume concepts in relation to modern imaging. While actual recommendations as the EORTC or ESTRO-ACROP guidelines already include imaging standards, the recently published PET-Plan trial prospectively investigated conventional versus imaging guided target volumes in relation to patient outcome. The results of this trial may help to further refine standards. The current review gives a practical overview on procedures for pre-treatment imaging and target volume delineation in locally advanced non-small cell lung cancer (NSCLC) in synopsis of the procedures established by the PET-Plan trial with the actual EORTC and ACROP guidelines.
\end{abstract}

Keywords: Non-small cell lung cancer (NSCLC); radiotherapy; target volume delineation

Submitted Jul 02, 2020. Accepted for publication Aug 04, 2020.

doi: $10.21037 /$ tlcr-20-805

View this article at: http://dx.doi.org/10.21037/tlcr-20-805

\section{Introduction}

In recent years, radiotherapy (RT) has been revolutionized technically by the implementation of highly precise treatment applications and increasingly conformal irradiation technology. In combination with modern systemic treatments like chemotherapy, targeted therapy and now also immunotherapy, this offers the opportunity for increased tumor control, less toxicity, and therefore improved outcomes for many cancer patients, including those with non-small cell lung cancer (NSCLC). Beyond the doses themselves, the beneficial effect of RT and RT dose escalation is dependent on a well-chosen target volume. Whilst the choice of a target volume may be relatively simple for small targets, e.g., in routine stereotactic RT, it becomes increasingly challenging in expanded locoregional treatment landscapes. Here, assumptions on microscopic 
spread must be made and the risk of tumor recurrence must be balanced against the risk of severe side effects to larger normal tissue volumes exposed. As known from prospective clinical trials, e.g., in head and neck or rectal cancer, adequate target volume delineation has an impact on tumor control and toxicity (1).

In many extra-thoracic tumors, larger target volumes result in bigger volumes of irradiated normal tissues with more or less tolerable but not necessarily life threatening side effects, therefore impairing long term quality of life. In the context of RT of locally advanced lung cancer, the definition of target volumes is crucially relevant, as both tumor progression and side effects of RT maybe the consequence of inadequate delineations and soon be life threatening to the patient. Due to the high radiation sensitivity of normal tissues in the chest, it is therefore a technical challenge to safely apply adequate RT treatment doses (2), while the poor outcome, of which beyond systemic spread local recurrence is one of the main issues (3), mandates more effective treatment $(4,5)$.

Although important, many RT target volume concepts are still based on old conventions, which rely on established practice or on clinical experience (e.g., on patterns of spread or recurrence) and on imaging standards from previous decades. In general, the translation of those old standards into three-dimensional (3D) target volumes for modern RT techniques has resulted in largely variable clinical practice, which has e.g. been demonstrated at the example of head and neck tumors (6). The gross tumor volume (GTV) may be clearer to define but is still a challenge in many cases. The variability largely furthermore affects clinical target volume (CTV) concepts, which are designed to cover assumed microscopic tumour spread. Due to the lack of prospective data, resulting guidelines often need to be based on consensus in this context.

Alongside the technical achievements in RT, large improvements have also been made in imaging. Molecular imaging methods such as positron-emission tomography (PET) have now been implemented, which in contrast to anatomical imaging, for example, computed tomography (CT), depict metabolic processes that are typical in certain aspects of tumor growth and are therefore of great interest in RT. For lung cancer, in recent years, PET/CT with 18F-fluoro-desoxy-glucose (FDG) has shown a superior diagnostic accuracy as compared to conventional imaging (7). It has therefore become an indispensable standard tool for diagnostic workup and staging and a useful modality for response assessment.
The current process of re-examining standards offers the chance to optimise target volume concepts in relation to modern imaging. With those, (I) the improved imaging of primary tumors and affected nodes could impact the delineation of the GTV and (II) improved accuracy in detecting or excluding the microscopic spread to neighboring tissues or nodes might inform new-potentially reduced-CTV concepts.

\section{Sources of current standards}

Due to the high clinical need, various initiatives have addressed the standardization of RT treatment planning and target volume delineation in locally advanced NSCLC. The series recommendations of the European Organization for Research on Treatment of Cancer (EORTC) on radiotherapy of NSCLC date back to 2004 (8) with a the last edition having been published in 2017 (9). The recommendations address pre-treatment workup including patient positioning, imaging and motion management, RT treatment planning including target volumes definition and dose constraints as well as treatment delivery for SBRT and fractionated RT of NSCLC and SCLC. The recommendations have evolved over time, always giving an up-to date view on current developments.

The ESTRO-ACROP committee is part of a quality initiative of the European SocieTy for Radiotherapy and Oncology (ESTRO), aiming at a standardization of RT procedures throughout Europe. The committee was initiated by the ESTRO to develop a guideline for target volume delineation in standard clinical scenarios like definitive (chemo)radiotherapy (RT) and adjuvant RT for locally advanced NSCLC. The group reviewed current literature and developed practice guidelines using a structured questionnaire followed by a consensus process with discussion and voting procedure. The resulting detailed recommendations (10) address diagnostics and imaging, treatment planning including imaging, target volume delineation, motion management, organs at risk as well as responsibilities and organization. In many points, this guideline is in line with the EORTC recommendations and beyond this gives detailed hints for daily practice.

Among the key literature, which has informed those recommendations, is the work of two expert groups organized by the International Atomic Energy Agency (IAEA), who reviewed literature and gave recommendations in recent years. In 2006 (11), an IAEA expert group reviewed the literature and provided evidence and recommendations 
on the use of FDG-PET in RT for NSCLC. The method had just become widely available and beyond its diagnostic advantages, it was clear that FDG-PET scans might be very useful in the context of RT-treatment planning. The experts identified a high potential of integrated recent FDG-PET/ CT in RT treatment position to be helpful in RT treatment planning. In follow up of this initiative, in 2014 (12), updated practice recommendations were given and the PERTAIN trial (13) was initiated, of which the preliminary results were published in 2019. In comparison with a historic cohort, the investigators showed an improvement of OS and PFS for advanced NSCLC by the introduction of quality controlled FDG-PET/CT guided treatment.

In 2008, another IAEA expert group addressed the question of elective nodal irradiation (ENI) in locally advanced NSCLC (14). Before this, somehow independent from the use of PET scans and in clear contrast to the current standards for other solid tumors, in the early 2000s, it had been proposed to omit ENI, i.e., the treatment of unaffected but neighboring nodal stations for suspected microscopic spread in favor for involved field radiotherapy (IFRT). In the light of some mainly retrospective evidence and due to the chance to reduce toxicity and escalate tumor doses by smaller RT volumes, the IAEA experts favored the omission of ENI and recommended prospective clinical trials on FDG-PET based dose escalation with volume reduction to investigate the benefit of the smaller volumes in terms of increased tumor control and/or reduced toxicity. The approach of omission of elective nodes was then supported by three randomized single-center trials from China (15-17), with no or limited PET scanning. A metaanalysis with the endpoint of elective nodal failure mainly based on these three studies and three more recent smaller cohort studies (of which one trial used PET scanning) did not show difference between IFRT and ENI (18).

In 2019, Schild et al. (19) published a pooled analysis investigating the impact of target volumes and treatment dose in a large cohort of patients, who participated in sixteen North American cooperative group trials of concurrent chemo-radiotherapy (CRT). The authors investigated, if RT field design strategy (ENI, mainly from more recent trials versus involved-field, IFRT, mainly from older trials) and dosing ( 60 versus $\geq 60 \mathrm{~Gy}$ ) were of relevance for toxicity and outcome. IFRT was associated with less, higher RT doses with more toxicity, and IFRT with 60 Gy was associated with more favorable overall survival and less toxicity than was ENI or higher RT doses. However, PET was required in only one more recent trial using IFRT and due to the lack of data, the relation of the use of PET scanning with outcome could not be assessed.

Still, although FDG-PET/CT is the most accurate non-invasive diagnostic method for staging of lung cancer presently available, which especially has a high negative predictive value (20). However, with a lower positive predictive value, its diagnostic accuracy is not perfect. Therefore, PET based RT-planning still bears a possible threat of impairing local or locoregional control by too excessive restriction of GTVs (21) and by non-irradiation of FDG-negative nodal spread (22) in too restricted CTVs. In the light of this limited evidence, the recently published PET-Plan trial (23) was initiated in 2006 to provide qualityassured prospective multicenter data assessing outcome and toxicity after the use of PET scans and the reduction of target volumes and dose escalation together.

In this prospective randomized trial, 205 patients were randomized from 24 centers in Germany, Austria and Switzerland. Patients with inoperable locally advanced NSCLC suitable for radio-chemotherapy having inoperable stage II or III tumors, suitable for CRT were included. In the conventional study arm A, target volumes were informed by FDG-PET and CT plus ENI. In the experimental study arm B they were informed by FDG-PET alone (Figure 1).

Other than in former trials mainly aiming at the safety of ENI, in this trial, investigating an even more restricted target volume concept, an isotoxic dose escalation between 60 and 74 Gy was performed and its primary endpoint was time to locoregional progression (LRP) with the objective to show non-inferiority of experimental arm B. As a result, the mean escalated total RT reference dose was significantly higher in the experimental arm B compared to arm A. For the primary endpoint LRP, non-inferiority of experimental arm B was confirmed and furthermore the risk of LRP in this study arm was nearly halved with no difference regarding overall survival, out-of-field recurrence and toxicity.

\section{Synopsis and update of recommendations}

From these sources, the following section gives a practical summary of current recommendations and shows the potential impact of the PET-Plan trial in on some crucial points of imaging, RT-preparation and GTV- and CTVdelineation of locally advanced NSCLC.

\section{Imaging and treatment preparation}

For preparation of curative-intent RT or chemo-RT 

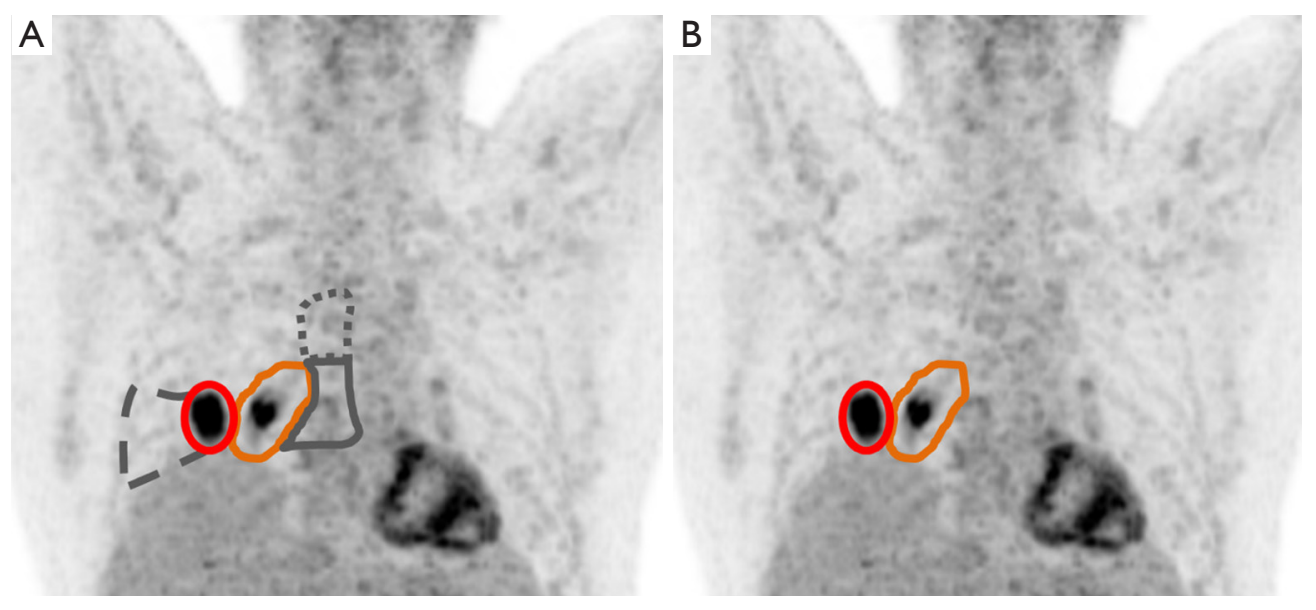

Figure 1 Schematic illustration of target volume delineation for radiochemotherapy of advanced NSCLC according to historic guidelines (A) in comparison to the PET-Plan system (B). (A) In historic guidelines, in addition to the FDG-PET positive tumor (red) and nodal areas (orange), if present, FDG-negative lymph node stations with nodes enlarged in CT (solid grey) and dys- or atelectasis (dashed grey) was included in the high-dose CTV. Furthermore, elective nodal stations, e.g., with a probability of involvement of $>10 \%$ (dotted grey) were historically treated. (B) According to the prospectively validated PET-Plan system, only an FDG-PET based CTV of tumor (red) and FDGpositive nodal areas (orange) will be targeted. NSCLC, non-small cell lung cancer; PET, positron-emission tomography; CT, computed tomography; FDG, 18F-fluoro-desoxy-glucose; CTV, clinical target volume.

in patients with NSCLC, according to the ACROP committee, a diagnostic CT scan with intravenous (IV) contrast and a diagnostic whole body FDG-PET-CT are considered mandatory, as FDG-PET has a higher diagnostic accuracy, when compared to CT alone (24) for the detection of lymph node metastases.

The acquisition protocol should undergo institutional standardization (25), when PET data are co-registered with anatomical imaging for RT planning. The FDG-PETCT scan should be performed within 3 weeks before start of treatment (12). PET-CT information may otherwise become outdated (26) with increasing time to treatment (27-29). As even one cycle of chemotherapy (30) can lead to a decrease in FDG-uptake, residual post-chemotherapy FDG-accumulations should not be used as the only source of information for the delineation of the gross tumour volume. When sequential chemotherapy is followed by definitive RT, it is highly recommended to perform a first FDG-PET-CT scan before induction chemotherapy and a repeat chest CT with IV iodine contrast prior to the start of RT. If this is not done, the pre-chemotherapyCT scan might inform GTV-delineation better than postchemotherapy-PET.

For the diagnosis of nodal involvement, additional tests should be considered beyond imaging. For radiologically enlarged lymph nodes (short axis $\geq 1 \mathrm{~cm}$ on the CT scan) or lymph nodes with increased focal FDG uptake on FDGPET-CT, biopsy (histology, cytology) is recommended, if it has impact on target volume definition.

In the context of the PET-Plan trial, PET-reading in preparation of RT-planning was investigated (31). An expert panel, which was prospectively installed performing blinded reviews of mediastinal NSCLC involvement in FDG-PET/ CT for all study patients, showed a high initial reporting inter-observer disagreement. Therefore, an interventional harmonization process was performed. After refinement of reading criteria, the agreement improved substantially and persistent. Subjective uncertainty was highly predictive for low agreement.

Reading variability was identified to be a major problem, possibly related to many factors, e.g., education, experience and the complexity of cases. This fact may lead to variable target volume delineations between individuals and centers, especially concerning the inclusion of nodal areas and mandates standardization. For this, joint discussion between radiation oncology and imaging with agreement on detailed reading and reporting criteria may be of help. As the subjective uncertainty of the observers is predictive for low inter-observer agreement, it should be communicated and included in decisions of critical parts of target volumes. In practice, while sensitive reading of PET scans in the context of restricted target volumes is highly recommended, 
uncertain findings in nodal areas with low pre-test probability, e.g., a contralateral hilum, can be excluded from the target volumes, if they would lead to compromises in applicable treatment doses and/or make curative intent treatment impossible.

Furthermore, a larger part of the disagreements was related to the varying anatomical allocation of given findings, the communication of pathologic findings by annotated images rather than by written reports, as it has been proposed by Bayne et al. (32) is therefore recommended.

\section{Planning-CT scan}

According to the ACROP and EORTC recommendations, a CT scan in treatment position is mandatory at the time of planning which should use IV iodine contrast to help delineation of the primary tumour and lymph node target volume and the organs at risk. The assessment of respiratory motion on a respiratory-correlated 4D-CT scan is recommended and its details are beyond the scope of this review.

The recommended specific planning-PET-CT scan should be done in RT-planning position. Here, according to EORTC and ACROP recommendations, the equipment used for patient immobilization should be identical between CT and PET scans scanning and treatment. Before delineation, the quality of the co-registration should be checked. The reason is, that patient movements may lead to incorrect hardware fusion, even when using a PET-CT machine. Caution is furthermore advised in using non-rigid registration algorithms (33), which have not been evaluated in the context of RT-planning. To avoid wrong co-registration with consecutive incorrect contouring, additional imaging datasets should not be coregistered in the treatment planning system when they have not been acquired in planning position. Those should rather be viewed side by side for selection of pathologic structures.

The PET-Plan procedures are basically in line with these recommendations. According of the experience of many study centers, ideally, the CT scan of the PET/CT should be used as Planning-CT.

\section{GTV definition}

According to both EORTC and ACROP recommendations, the delineation of the GTV should be based on both recent $\mathrm{CT}$ and FDG-PET information. As the measured diameter of tumors in lung or mediastinum is dependent on the display window chosen to analyze CT slices (34), CT-based delineation with standardized window settings is recommended.

Here, the GTV of the primary tumor and nodal GTVs should be drawn separately, if anatomically distinguishable. The best concordance between measured and actual diameters and volumes for CT will be reached with the settings: $W=1,600$ and $\mathrm{L}=600 \mathrm{HU}$ (Hounsfield Units) for parenchyma, and W $=400$ and $\mathrm{L}=20 \mathrm{HU}$ (Hounsfield Units) for mediastinum. This lung window setting should be used to delineate tumors surrounded by lung tissue while the mediastinum window setting for the delineation of lymph nodes and primary tumors invading the mediastinum or chest wall.

For the use of FDG-PET scans for GTV-delineation, the following recommendations apply: a simple and widely used approach for FDG based target volume definition is visual contouring at standardized window setting with a clinical protocol that integrates all relevant information together with the reports of the nuclear medicine physician and radiologist (33). Even when PET is co-registered with CT, any approach other than that should be used with caution in experienced centers. When there automatic contouring algorithms are used (even "simple" SUV- or percent-thresholding), those should be calibrated and their results should always be validated in clinical routine, e.g., versus well visible findings in $\mathrm{CT}$.

In the PET-Plan trial, e.g., the implementation of a standardized individually calibrated contrast-oriented semi-automatic contouring algorithm $(35,36)$, which had undergone multi-center calibration (37), was encouraged and it was used for the majority of cases as a starting point for GTV-contouring. Enlargement, e.g., by CT information, but not reduction of this volume by the user was allowed. In the other cases, visual contouring in cooperation between radiation oncology and diagnostic imaging colleagues was performed.

Up to the publication of recent EORTC recommendations and the ACROP guideline, the use of FDG-PET scans to differentiate tumour from atelectasis has not been subject to pathological or clinical studies. However, due to clinical plausibility it was recommended that regions of atelectasis visible on the CT image beyond the edge of the increased FDG uptake may be excluded from the GTV (12). In the PET-Plan trial, one of the differences between the study arms was the in- vs. exclusion of tumor-associated atelectasis. Concluded from the non-inferiority of the experimental study arm B (where 
atelectasis beyond the FDG-based GTV was not part of the target volume) and no difference seen between study arms in terms of out-field recurrences, there is now additional evidence supporting this recommendation.

Generally, the delineation of nodal GTVs is recommended in the process of target volume delineation. According to ACROP recommendations, accurate delineation and correct identification of the anatomy is well supported by the use of a CT scan with IV contrast. Beyond nodes that are enlarged in CT (update: see below), lymph nodes proven malignant by biopsy or considered pathological on PET (focal accumulation above blood pool) are delineated as GTV. Accounting for the inter-observer variation in the reporting of FDGpositive mediastinal nodes, in equivocal cases, a node should rather be included than excluded in the GTV (see above). Lymph nodes that are FDG-PET-positive and negative by endoscopic bronchial ultrasound (EBUS) or endoscopic esophageal ultrasound (EUS) should be included in the GTV due to the fact that the false negative rates of EBUS/EUS are relatively high (22). PET positive nodes may be omitted, if there is clear nonmalignant biopsy explanation for the FDG positivity $(22,38)$.

With regard to the results of the PET-Plan trial, no difference in the rate of out-field recurrences was seen, when nodes, that were enlarged by CT but FDGnegative were not included in the target volume. As smaller treatment volumes may improve local control or reduce toxicity, this approach, more restrictive than former recommendations, should be followed.

Concerning the situation, that a GTV may be drawn after chemotherapy, care must be taken. In FDG-PET/ $\mathrm{CT}$, false negative findings may lead to geographic miss and an undertreatment of vital tumor tissue. Here the ACROP committee recommend that the GTV of the primary tumor after induction chemotherapy should be based on current CT imaging. For information, pre-chemotherapy imaging (including PET-CT) should also be considered. The EORTC recommendations propose that the use of coregistered pre-treatment and planning $\mathrm{CT}$ and/or PETCT scans can enable a more accurate reconstruction of prechemotherapy target volumes (39).

Post-chemotherapy nodal GTVs should therefore include all involved lymph nodes or lymph node stations based on pre-chemotherapy clinical, pathological and imaging information, even if a node has completely disappeared in imaging.

In the PET-Plan trial, induction chemotherapy was an exclusion criterion, so that all volumes delineated here were derived from primary PET/CT scans.

\section{CTV definition}

\section{Primary tumor}

According to EORTC recommendations, a CTV margin around the primary tumor and lymph should be tailored according to the histology of the primary tumor (40), size of lymph node (41) and possibly, imaging characteristics of the tumor (42). The ACROP committee recommends that the CTV of the primary tumor should be created by an expansion from the GTV (e.g., 5-8 mm) (40) and manually edited for surrounding anatomy, e.g., natural barriers such as bones or heart $(41,43)$.

In the PET-Plan trial, the FDG-based GTV was expanded into all directions by $2 \mathrm{~mm}$. The rationale for the size of this margin was, that the rather generous interpretation of the PET signal (being derived from 3D PET) by the algorithm used, did include some effects of movement blurring $(44,45)$.

\section{Nodal CTVs}

Generally, all sources recommend that when delineating nodal stations, an atlas, e.g., the TNM Atlas 8th edition should be used as a basis for lymph node region definition. Ideally, the institutional standard should use a $3 \mathrm{D}$ illustration (46).

For the delineation nodal CTVs, the ACROP committee discussed two options:

Option 1 includes the whole anatomically defined lymph node stations. Practically, it is the inclusion of the whole pathologically affected lymph node station including at least a 5-8 $\mathrm{mm}$ margin around the nodal GTV. This option has been used in the PET-Plan trial. Its safety as to outfield recurrences has therefore been prospectively shown.

Option 2 means the geometric expansion of the nodal GTV. Practically, the nodal GTV is expanded to the CTV in analogy to the primary tumor $(5-8 \mathrm{~mm})$. As described above, the margin may be tailored according to the size of lymph nodes or histology of the primary tumor (see above) but care should be taken with respect to neighboring normal organs (e.g., esophagus) in order to not increase toxicity. Although this approach may somehow appear logical, its safety has not been prospectively tested. A source 
of error may especially lie in interpretation of inconclusive findings, which is well illustrated by the above-mentioned panel harmonization process of the PET-Plan trial. Beyond reading differences, a large percentage of incongruent imaging reports in this analysis were found to be caused by anatomical discrepancies. Therefore, this option should only be used by experts or groups, who are well experienced in the interpretation of CT and FDG-PET images and who have a well-established communication between imaging and radiation oncology about those.

As to the question of ENI, the EORTC group recommends that ENI is not indicated in any patient group that receives curative or radical doses of RT for inoperable NSCLC $(47,48)$. The ACROP committee adds that beyond nodal CTVs as cited above, elective inclusion of the hilum and/or neighboring nodal lymph node stations can be considered. Inclusion of uninvolved areas between involved stations (especially the hilum) is optional.

Here, the PET-Plan trial leads to an update of evidence. By restricting nodal CTVs to the FDG-PET positive nodal stations only ENI does not lead to an improved outcome nor to more safety towards out-field recurrence or toxicity. It should therefore be omitted.

\section{Summary delineation procedure}

As to the PET-Plan procedures, the following process for GTV and CTV delineation may be followed in locally advanced NSCLC (see also: Table 1, Figure 1):

Firstly, the GTV of the primary tumor should be delineated based on recent FDG-PET in a well coregistered planning CT, the use of a locally validated semiautomatic algorithm may ease this process $(36,37)$. Manual adjustments to enlarge but not reduce the tumor contour according to CT morphology are possible, but FDG-negative atelectasis should not be included. Then, this GTV should be expanded into all directions by at least $2 \mathrm{~mm}$. The institutional rationale for the size of the GTVCTV-margin may depend on the interpretation of the PET signal, as being derived from well coregistered 3D PET, it may include some effects of movement blurring.

For nodal CTVs, FDG-positive LNs (according to sensitive reading) should be allocated to the respective Mountain-Dresler LN levels and those should be delineated in accordance with a contouring atlas. Respect should be taken to any anatomic changes, e.g., when grossly enlarged nodes exceed the boundaries of atlas structures. LN levels, which are not involved according to PET but confirmed by biopsy, should also be included in the CTV.

\section{Radiotherapy quality assurance (RTQA)}

In the context of recent trials, namely after the unexpected results of the RTOG 0617 trial, the question of RTQA impacting tumor control and normal tissue toxicity was increasingly put into attention. Several groups have addressed the importance of heart doses in this context, another question may be PTV coverage when trying to achieve dose escalation. As a negative effect of higher doses was not seen in the analysis of the PET-Plan trial so far, this may have been related to the rigorous QA programme of that trial, which led to encouragingly low rate of RTQA noncompliance as compared to literature. The standardization addressed imaging and planning procedures $(37,49)$ FDG-PET/CT scan reading for use in RT planning (31), physical RT planning and application, and reading of follow-up imaging. With increasing complexity of treatment technologies, there is also an increasing role of institutional and multicenter QA for good RT practice. This importance is also addressed by the ACROP committee recommending the development of institutional standards, hands-on training and a peer-reviewed quality assurance process.

\section{Open questions and new developments}

\section{Growing importance of volume independent from dose in times of immunotherapy?}

The PET-Plan trial was designed around the same time and conducted in a similar population to the RTOG 0617 trial, although the focus was different. In RTOG 0617 (50), combined CRT (partly with cetuximab) and high RT doses of $74 v s$. 60 Gy did not lead to improved outcome. Dose range and normal tissue constraints were comparable between both trials. However, in the setting of PETPlan, individual investigators were not obligated to give the highest dose to pre-defined patients, but could decide on the highest plannable dose while keeping mandatory normal tissue constraints. Other differences between both trials involve the systemic treatment component used and imaging in follow up. Overall, in contrast to RTOG 0617, in the PET-Plan trial, negative effects of high RT treatment doses on survival or toxicity were not observed. However, although an improved local control in the experimental arm was noted, it could not be demonstrated that this effect was positively related to dose.

The retrospective analysis of Schild et al. (19) also showed improved local control in reduced target volumes not necessarily related to dose. So, it may be, that other 
Table 1 Practical tips for FDG-PET based target volume contouring in locally advanced NSCLC

Do only coregister a PET/CT scan for contouring in a planning system, if it was acquired in RT treatment position. If not, view images separately side by side

Check co-registration of PET and CT before planning

Be careful: systemic treatment like chemotherapy may lead to false negative findings in FDG-PET

The PET-scan should be read sensitive (i.e., equivocal lesions should be regarded as tumor, especially in high-risk-regions) when used to design FDG-PET restricted target volumes

Do not include FDG-negative atelectasis

Do not include FDG-negative enlarged lymph nodes, unless pathologically positive

GTV-contours derived from PET should not be smaller than those generated by CT, unless tumor or nodes are not clearly visible there, e.g., because of atelectasis

When contouring in 3D-PET scan, blurring might be included in PET-contour and CTV margins may then be reduced to, e.g., 2 mm. Check this with findings, that can be seen both in CT and PET

If automatic contouring is applied, use locally validated method, otherwise do manual contouring together with imaging specialist

For nodal CTV-volumes it is the safer option to include whole anatomically defined nodal stations. If including pathologic nodes only, make sure that decision is diagnostically safe and apply adequate CTV-margin

NSCLC, non-small cell lung cancer; PET, positron-emission tomography; CT, computed tomography; FDG, 18F-fluoro-desoxy-glucose; GTV, gross tumor volume; CTV, clinical target volume.

reasons like volume size or location are involved. With today's knowledge about tumor-immuno-interactions, one may discuss, if target volume restriction, independently from treatment dose, may be beneficial, as long as the macroscopic tumor and affected nodes are safely targeted. One may speculate that a reduction of CTVs could contribute to optimum RT-induced immune stimulation by omitting the irradiation of draining lymph nodes (51). A hint towards this may be, that it has been shown in the context of stereotactic radiotherapy (SBRT) - somehow an extreme example of target volume restriction-that the experimental addition of mediastinal irradiation led to unfavorable immune effects (51). It has further been shown in esophageal cancer, that the extent of lymphopenia, which was related to mean body radiation exposure, is predictive for the results of radiochemotherapy (52). Thereforebeyond normal tissue toxicity reduction-target volume concepts which reduce irradiation of unaffected normal tissues may have beneficial immune-effects, especially when they contain immunologically important structures.

This may become an even more crucial issue with new combination therapies as recently improved overall survival by adding the PD-L1 inhibitor durvalumab after concurrent CRT has been shown (53). With an improved systemic treatment component, progression of macroscopic tumor still remains a threat and therefore an optimum local treatment component for inoperable patients is important to further improve outcomes. On the other side, along with the increasing potential of systemic treatment to treat microscopic spread, the concentration of RT on the macroscopic tumor makes increasing sense. As beneficial effects of immunotherapy are also seen for operable patients with more being awaited, adjuvant and neoadjuvant RT will also be facing changes in the future. However, this is beyond the scope of our review but more information can be found in the article on immunogenic cell death in this issue of TLCR.

\section{Beyond lung cancer}

The concept of imaging-based target volume restriction may not only be of interest for lung cancer patients. In cancer survivors, although not life-threatening, late RT toxicity significantly impairs quality of life, e.g., after definitive RT treatment of head and neck (54) or pelvic tumors (55). Late normal tissue effects may not only be related to irradiation of the tumor site itself but also to relatively large traditional CTVs intended to cover microscopic spread. As oncologically favorable outcomes are often seen despite considerable disagreement about appropriate CTVs (6), some parts of those may not be required and some late toxicity may therefore be avoidable. 
Prospective trials on imaging-based target volume restriction will enhance progress in this field.

However, optimum imaging may not always be FDGPET or CT. For every tumor and clinical scenario, for revision of CTV concepts, the imaging literature must be reviewed for diagnostic data, which are robust enough to inform target volume reduction. Whilst in NSCLC, FDGPET/CT presently appears to be the optimal method, this may not be the case in other tumors like prostate cancer or glioma, where other methods, such as multi-parametric MRI and PET/CT with prostate-specific membrane antigen (PSMA) (56) or amino acid PET (57) may be the imaging tools of choice.

\section{Acknowledgments}

Funding: None.

\section{Footnote}

Provenance and Peer Review: This article was commissioned by the Guest Editors (Jacek Jassem and Rafal Dziadziuszko) for the series "Radiotherapy in thoracic malignancies" published in Translational Lung Cancer Research. The article was sent for external peer review organized by the Guest Editors and the editorial office.

Peer Review File: Available at http://dx.doi.org/10.21037/ tlcr-20-805

Conflicts of Interest: All authors have completed the ICMJE uniform disclosure form (available at http://dx.doi. org/10.21037/tlcr-20-805). The series "Radiotherapy in thoracic malignancies" was commissioned by the editorial office without any funding or sponsorship. The authors have no other conflicts of interest to declare.

Ethical Statement: The authors are accountable for all aspects of the work in ensuring that questions related to the accuracy or integrity of any part of the work are appropriately investigated and resolved.

Open Access Statement: This is an Open Access article distributed in accordance with the Creative Commons Attribution-NonCommercial-NoDerivs 4.0 International License (CC BY-NC-ND 4.0), which permits the noncommercial replication and distribution of the article with the strict proviso that no changes or edits are made and the original work is properly cited (including links to both the formal publication through the relevant DOI and the license). See: https://creativecommons.org/licenses/by-nc-nd/4.0/.

\section{References}

1. Weber DC, Hurkmans CW, Melidis C, et al. Outcome impact and cost-effectiveness of quality assurance for radiotherapy planned for the EORTC 22071-24071 prospective study for head and neck cancer. Radiother Oncol 2014;111:393-9.

2. Kong FM, Ritter T, Quint DJ, et al. Consideration of dose limits for organs at risk of thoracic radiotherapy: atlas for lung, proximal bronchial tree, esophagus, spinal cord, ribs, and brachial plexus. Int J Radiat Oncol Biol Phys 2011;81:1442-57.

3. Machtay M, Paulus R, Moughan J, et al. Defining local-regional control and its importance in locally advanced non-small cell lung carcinoma. J Thorac Oncol 2012;7:716-22.

4. Saunders M, Dische S, Barrett A, et al. Continuous, hyperfractionated, accelerated radiotherapy (CHART) versus conventional radiotherapy in non-small cell lung cancer: mature data from the randomised multicentre trial. CHART Steering committee. Radiother Oncol 1999;52:137-48.

5. Walraven I, van den Heuvel M, van Diessen J, et al. Longterm follow-up of patients with locally advanced non-small cell lung cancer receiving concurrent hypofractionated chemoradiotherapy with or without cetuximab. Radiother Oncol 2016;118:442-6.

6. Hong TS, Tome WA, Harari PM. Heterogeneity in head and neck IMRT target design and clinical practice. Radiother Oncol 2012;103:92-8.

7. Ambrosini V, Nicolini S, Caroli P, et al. PET/CT imaging in different types of lung cancer: an overview. Eur J Radiol 2012;81:988-1001.

8. Senan S, De Ruysscher D, Giraud P, et al. Literature-based recommendations for treatment planning and execution in high-dose radiotherapy for lung cancer. Radiother Oncol 2004;71:139-46.

9. De Ruysscher D, Faivre-Finn C, Moeller D, et al. European Organization for Research and Treatment of Cancer (EORTC) recommendations for planning and delivery of high-dose, high precision radiotherapy for lung cancer. Radiother Oncol 2017;124:1-10.

10. Nestle U, De Ruysscher D, Ricardi U, et al. ESTRO ACROP guidelines for target volume definition in the 
treatment of locally advanced non-small cell lung cancer. Radiother Oncol 2018;127:1-5.

11. MacManus M, Nestle U, Rosenzweig KE, et al. Use of PET and PET/CT for radiation therapy planning: IAEA expert report 2006-2007. Radiother Oncol 2009;91:85-94.

12. Konert T, Vogel W, MacManus MP, et al. PET/CT imaging for target volume delineation in curative intent radiotherapy of non-small cell lung cancer: IAEA consensus report 2014. Radiother Oncol 2015;116:27-34.

13. Konert T, Vogel WV, Paez D, et al. Introducing FDG PET/CT-guided chemoradiotherapy for stage III NSCLC in low- and middle-income countries: preliminary results from the IAEA PERTAIN trial. Eur J Nucl Med Mol Imaging 2019;46:2235-43.

14. Belderbos JS, Kepka L, Spring Kong FM, et al. Report from the International Atomic Energy Agency (IAEA) consultants' meeting on elective nodal irradiation in lung cancer: non-small-Cell lung cancer (NSCLC). Int J Radiat Oncol Biol Phys 2008;72:335-42.

15. Yuan S, Sun X, Li M, et al. A randomized study of involved-field irradiation versus elective nodal irradiation in combination with concurrent chemotherapy for inoperable stage III nonsmall cell lung cancer. Am J Clin Oncol 2007;30:239-44.

16. Yang K, Cao F, Wang J, et al. Improved local control without elective nodal radiotherapy in patients with unresectable NSCLC treated by 3D-CRT. Front Med China 2007;1:381-5.

17. Chen M, Bao Y, Ma HL, et al. Involved-field radiotherapy versus elective nodal irradiation in combination with concurrent chemotherapy for locally advanced non-small cell lung cancer: a prospective randomized study. Biomed Res Int 2013;2013:371819.

18. Li R, Yu L, Lin S, et al. Involved field radiotherapy (IFRT) versus elective nodal irradiation (ENI) for locally advanced non-small cell lung cancer: a meta-analysis of incidence of elective nodal failure (ENF). Radiat Oncol 2016;11:124.

19. Schild SE, Fan W, Stinchcombe TE, et al. Toxicity Related to Radiotherapy Dose and Targeting Strategy: A Pooled Analysis of Cooperative Group Trials of Combined Modality Therapy for Locally Advanced Non-Small Cell Lung Cancer. J Thorac Oncol 2019;14:298-303.

20. Schmidt-Hansen M, Baldwin DR, Hasler E, et al. PET-CT for assessing mediastinal lymph node involvement in patients with suspected resectable nonsmall cell lung cancer. Cochrane Database Syst Rev 2014;2014:CD009519.

21. Sura S, Greco C, Gelblum D, et al. (18)
F-fluorodeoxyglucose positron emission tomographybased assessment of local failure patterns in non-smallcell lung cancer treated with definitive radiotherapy. Int J Radiat Oncol Biol Phys 2008;70:1397-402.

22. Peeters ST, Dooms C, Van Baardwijk A, et al. Selective mediastinal node irradiation in non-small cell lung cancer in the IMRT/VMAT era: How to use E(B)USNA information in addition to PET-CT for delineation? Radiother Oncol 2016;120:273-8.

23. Nestle U, Schimek-Jasch T, Kremp S, et al. Imagingbased target volume reduction in chemoradiotherapy for locally advanced non-small-cell lung cancer (PET-Plan): a multicentre, open-label, randomised, controlled trial. Lancet Oncol 2020;21:581-92.

24. Janssens G, Jacques L, Orban de Xivry J, et al. Diffeomorphic registration of images with variable contrast enhancement. Int J Biomed Imaging 2011;2011:891585.

25. van Herk M, Remeijer $P$, Rasch $C$, et al. The probability of correct target dosage: dose-population histograms for deriving treatment margins in radiotherapy. Int J Radiat Oncol Biol Phys 2000;47:1121-35.

26. Everitt S, Herschtal A, Callahan J, et al. High rates of tumor growth and disease progression detected on serial pretreatment fluorodeoxyglucose-positron emission tomography/computed tomography scans in radical radiotherapy candidates with nonsmall cell lung cancer. Cancer 2010;116:5030-7.

27. Everitt SJ, Ball DL, Hicks RJ, et al. Differential 18F-FDG and 18F-FLT uptake on serial PET/CT imaging before and during definitive chemoradiation for non-small cell lung cancer. J Nucl Med 2014;5 5:1069-74.

28. Geiger GA, Kim MB, Xanthopoulos EP, et al. Stage migration in planning PET/CT scans in patients due to receive radiotherapy for non-small-cell lung cancer. Clin Lung Cancer 2014;15:79-85.

29. Booth K, Hanna GG, McGonigle N, et al. The mediastinal staging accuracy of $18 \mathrm{~F}$-Fluorodeoxyglycose Positron Emission Tomography/Computed Tomography in non-small cell lung cancer with variable time intervals to surgery. Ulster Med J 2013;82:75.

30. Weber WA, Petersen V, Schmidt B, et al. Positron emission tomography in non-small-cell lung cancer: prediction of response to chemotherapy by quantitative assessment of glucose use. J Clin Oncol 2003;21:2651-7.

31. Nestle U, Rischke HC, Eschmann SM, et al. Improved inter-observer agreement of an expert review panel in an oncology treatment trial--Insights from a structured interventional process. Eur J Cancer 2015;51:2525-33. 
32. Bayne M, Hicks RJ, Everitt S, et al. Reproducibility of "intelligent" contouring of gross tumor volume in non-small-cell lung cancer on PET/CT images using a standardized visual method. Int J Radiat Oncol Biol Phys 2010;77:1151-7.

33. Thorwarth D, Beyer T, Boellaard R, et al. Integration of FDG-PET/CT into external beam radiation therapy planning: technical aspects and recommendations on methodological approaches. Nuklearmedizin 2012;51:140-53.

34. Harris KM, Adams H, Lloyd DC, et al. The effect on apparent size of simulated pulmonary nodules of using three standard CT window settings. Clin Radiol 1993;47:241-4.

35. Nestle U, Kremp S, Schaefer-Schuler A, et al. Comparison of different methods for delineation of 18F-FDG PETpositive tissue for target volume definition in radiotherapy of patients with non-Small cell lung cancer. J Nucl Med 2005;46:1342-8.

36. Schaefer A, Kremp S, Hellwig D, et al. A contrast-oriented algorithm for FDG-PET-based delineation of tumour volumes for the radiotherapy of lung cancer: derivation from phantom measurements and validation in patient data. Eur J Nucl Med Mol Imaging 2008;35:1989-99.

37. Schaefer A, Nestle U, Kremp S, et al. Multi-centre calibration of an adaptive thresholding method for PETbased delineation of tumour volumes in radiotherapy planning of lung cancer. Nuklearmedizin 2012;51:101-10.

38. Steinfort DP, Siva S, Leong TL, et al. Systematic endobronchial ultrasound-guided mediastinal staging versus positron emission tomography for comprehensive mediastinal staging in NSCLC before radical radiotherapy of non-small cell lung cancer: a pilot study. Medicine (Baltimore) 2016;95:e2488.

39. Lagerwaard FJ, van de Vaart PJ, Voet PW, et al. Can errors in reconstructing pre-chemotherapy target volumes contribute to the inferiority of sequential chemoradiation in stage III non-small cell lung cancer (NSCLC)? Lung Cancer 2002;38:297-301.

40. Giraud P, Antoine M, Larrouy A, et al. Evaluation of microscopic tumor extension in non-small-cell lung cancer for three-dimensional conformal radiotherapy planning. Int J Radiat Oncol Biol Phys 2000;48:1015-24.

41. Yuan S, Meng X, Yu J, et al. Determining optimal clinical target volume margins on the basis of microscopic extracapsular extension of metastatic nodes in patients with non-small-cell lung cancer. Int J Radiat Oncol Biol Phys 2007;67:727-34.
42. Berthelot K, Thureau S, Giraud P. Margin determination from clinical to planning target volume for lung cancer treated with conformal or intensity-modulated irradiation. Cancer Radiother 2016;20:616-21.

43. van Diessen JN, Chen C, van den Heuvel MM, et al. Differential analysis of local and regional failure in locally advanced non-small cell lung cancer patients treated with concurrent chemoradiotherapy. Radiotherapy and Oncology 2016;118:447-52.

44. Siedschlag C, Boersma L, van Loon J, et al. The impact of microscopic disease on the tumor control probability in non-small-cell lung cancer. Radiother Oncol 2011;100:344-50.

45. van Loon J, Siedschlag C, Stroom J, et al. Microscopic disease extension in three dimensions for non-small-cell lung cancer: development of a prediction model using pathology-validated positron emission tomography and computed tomography features. Int J Radiat Oncol Biol Phys 2012;82:448-56.

46. Lynch R, Pitson G, Ball D, et al. Computed tomographic atlas for the new international lymph node map for lung cancer: A radiation oncologist perspective. Pract Radiat Oncol 2013;3:54-66.

47. De Ruysscher D, Wanders S, van Haren E, et al. Selective mediastinal node irradiation based on FDG-PET scan data in patients with non-small-cell lung cancer: a prospective clinical study. Int J Radiat Oncol Biol Phys 2005;62:988-94.

48. Belderbos JS, Heemsbergen WD, De Jaeger K, et al. Final results of a Phase I/II dose escalation trial in non-small-cell lung cancer using three-dimensional conformal radiotherapy. Int J Radiat Oncol Biol Phys 2006;66:126-34.

49. Schimek-Jasch T, Troost EG, Rucker G, et al. A teaching intervention in a contouring dummy run improved target volume delineation in locally advanced non-small cell lung cancer: Reducing the interobserver variability in multicentre clinical studies. Strahlenther Onkol 2015;191:525-33.

50. Bradley JD, Paulus R, Komaki R, et al. Standard-dose versus high-dose conformal radiotherapy with concurrent and consolidation carboplatin plus paclitaxel with or without cetuximab for patients with stage IIIA or IIIB non-small-cell lung cancer (RTOG 0617): a randomised, two-by-two factorial phase 3 study. Lancet Oncol 2015;16:187-99.

51. Marciscano AE, Ghasemzadeh A, Nirschl TR, et al. Elective Nodal Irradiation Attenuates the Combinatorial 
Efficacy of Stereotactic Radiation Therapy and Immunotherapy. Clin Cancer Res 2018;24:5058-71.

52. Davuluri R, Jiang W, Fang P, et al. Lymphocyte Nadir and Esophageal Cancer Survival Outcomes After Chemoradiation Therapy. Int J Radiat Oncol Biol Phys 2017;99:128-35.

53. Antonia SJ, Villegas A, Daniel D, et al. Overall Survival with Durvalumab after Chemoradiotherapy in Stage III NSCLC. N Engl J Med 2018;379:2342-50.

54. van der Molen L, van Rossum MA, Burkhead LM, et al. Functional outcomes and rehabilitation strategies in patients treated with chemoradiotherapy for advanced head and neck cancer: a systematic review. Eur Arch Otorhinolaryngol 2009;266:889-900.

Cite this article as: Nestle U, Le Pechoux C, De Ruysscher D. Evolving target volume concepts in locally advanced non-small cell lung cancer. Transl Lung Cancer Res 2021;10(4):19992010. doi: $10.21037 /$ tlcr-20-805
55. Dearnaley D, Griffin CL, Lewis R, et al. Toxicity and Patient-Reported Outcomes of a Phase 2 Randomized Trial of Prostate and Pelvic Lymph Node Versus Prostate only Radiotherapy in Advanced Localised Prostate Cancer (PIVOTAL). Int J Radiat Oncol Biol Phys 2019;103:605-17.

56. Zamboglou C, Drendel V, Jilg CA, et al. Comparison of (68)Ga-HBED-CC PSMA-PET/CT and multiparametric MRI for gross tumour volume detection in patients with primary prostate cancer based on slice by slice comparison with histopathology. Theranostics 2017;7:228-37.

57. Grosu AL, Weber WA. PET for radiation treatment planning of brain tumours. Radiother Oncol 2010;96:325-7. 